Mathematical Modelling and Analysis

Volume 19 Number 4, September 2014, 491-508

http://dx.doi.org/10.3846/13926292.2014.956354

(c) Vilnius Gediminas Technical University, 2014
Publisher: Taylor\&Francis and VGTU

http://www.tandfonline.com/TMMA

Print ISSN: 1392-6292

Online ISSN: 1648-3510

\title{
A Class of Quasistatic Contact Problems for Viscoelastic Materials with Nonlocal Coulomb Friction and Time-Delay*
}

\author{
Si-sheng Yao $^{a, b}$ and Nan-jing Huang $^{b}$ \\ ${ }^{a}$ Department of Mathematics, Kunming University \\ Kunming, 650221 Yunnan, China \\ ${ }^{b}$ Department of Mathematics, Sichuan University \\ Chengdu, 610064 Sichuan, China \\ E-mail(corresp.): nanjinghuang@hotmail.com \\ E-mail: yaosisheng@outlook.com
}

Received July 8, 2013; revised July 4, 2014; published online September 1, 2014

\begin{abstract}
In this paper, a mathematical model which describes the explicit time dependent quasistatic frictional contact problems is introduced and studied. The material behavior is described with a nonlinear viscoelastic constitutive law with time-delay and the frictional contact is modeled with nonlocal Coulomb boundary conditions. A variational formulation of the mathematical model is given, which is called a quasistatic integro-differential variational inequality. Using the Banach's fixed point theorem, an existence and uniqueness theorem of the solution for the quasistatic integro-differential variational inequality is proved under some suitable assumptions. As an application, an existence and uniqueness theorem of the solution for the dual variational formulation is also given.
\end{abstract}

Keywords: quasistatic variational inequality, viscoelastic material, time-delay, nonlocal Coulomb friction law.

AMS Subject Classification: 49J40; 74D10.

\section{Introduction}

Viscoelastic constitutive laws have been used in the engineering or mathematical literature to describe the deformed behavior of contact problems. $\mathrm{Li}$ and $\mathrm{Xu}$ [14] discussed a process of hysteresis loop and energy dissipation of viscoelastic solid models. In 2005, Barboteu et al. [2] studied a class of abstract evolutionary variational inequalities arising in the study of contact problems for viscoelastic materials. Barboteu et al. [3] considered a frictionless viscoelastic

* This work was supported by the Key Program of NSFC (Grant No. 70831005), the National Natural Science Foundation of China (11171237) and the Scientific Research Foundation of Yunnan Provincial Education Office (2010Z024). 
piezoelectric contact problem. In this paper, they gave some numerical analysis results for the variational problem. In the framework of viscoelastic model, Argatov [1] used the asymptotic models for low and high rate loading to study the articular cartilage layer. Denkowski et al. [9] discussed the dynamic bilateral frictional contact problem. And the further Mathematical analysis and numerical approximation in connection with the contact problems can be found in the books $[12,15,18]$, and in the special issue [21].

When the forces applied to a frictional contact system vary slowly in time, the problem can be considered as quasistatic, in which the acceleration is negligible and the effect of temperature changes due to energy dissipation on the deformation of the material is negligible, too. Recently, some kinds of quasistatic frictional contact problems with viscoelastic materials have been considered and investigated by several authors. By using arguments for time dependent elliptic variational inequalities and fixed point theorem, Chau et al. [5] proved some existence and uniqueness results for quasistatic problem and showed that the solution of the viscoelastic problem converges to the solution of the relevant elastic problem. Delost and Fabre [8] presented a valid approximation method for a quasistatic abstract variational inequality with time independent constraint, and applied these results to the approximation of the quasistatic evolution of an elastic contact problem. The framework of quasistatic frictional contact problems was constructed by Han and Sofonea in the book [12], in which they provided the relevant mathematical analysis and numerical approximation. For more work concerned with quasistatic contact problems, we refer to $[11,23]$ and the references there in.

Elastic or viscoelastic contact problems with explicit time dependent operators were investigated in a large number of papers. Using the theory of evolutionary hemivariational inequality, Migórski et al. [16] proved that a nonlinear explicit time dependent elastic-viscoplastic frictional contact problem exists a unique weak solution. In [17], Migórski et al. considered a class of quasistatic contact models which describe the explicit time dependent viscoelastic problems. Based on the fixed point theorem for multi-valued mappings and variational-hemivariational inequality theory, Costea and Matei [7] proved the existence of weak solution for the general and unified framework contact models. They also discussed the uniqueness, the boundedness and the stability of the weak solution under some suitable conditions. In [13], Kulig and Migórski dealt with a class of second order nonlinear evolution inclusions with explicit time dependent operators and provided a theorem on the continuous dependence of the solution to the inclusion with respect to the operators involved in the problem.

Time-delay phenomena are frequently encountered in various technical systems, such as electric, pneumatic, hydraulic networks, and chemical processes. Moreover, variational inequalities with time-delay have become a vital part of optimal control problems. The description of models about time-delay phenomena can be found in $[19,26]$ and the general results of variational inequalities with time-delay can be found in $[6,25]$. However, to the best of our knowledge, there are only a few papers to study contact problems for viscoelastic materials with time-delay. In the recent paper [24], Yao and Huang introduced 
and studied a mathematical model which describes an explicit time-dependent quasistatic frictional contact problem between a deformable body and a foundation, in which the contact is bilateral, the behavior of the material is described with a viscoelastic constitutive law with time-delay, and the friction is modeled with Tresca's friction law with the friction bound depending on the total slip.

Motivated and inspired by the work mentioned above, in this paper, we introduce and study a class of quasistatic problems for a mathematical model of frictional contact between a deformable body and a foundation. The body is assumed to have a nonlinear behavior, the friction is modeled with nonlocal Coulomb friction law and the behavior of the body is described with a viscoelastic constitutive law with time-delay. We give a variational formulation of the problem which is called a quasistatic integro-differential variational inequality. By using the Banach's fixed-point theorem, we establish an existence and uniqueness theorem of the solution to the quasistatic contact problems for viscoelastic materials with nonlocal Coulomb friction and time-delay. As an application, we show an existence and uniqueness theorem of the solution for the dual variational formulation. The results presented in this paper generalize and improve some known results in [12] and [22].

\section{Preliminaries}

Let $d$ be a positive integer and $\mathcal{R}^{d}$ be a $d$-dimensional Euclidean space. Let $\mathcal{S}^{d}$ denote the space of all second order symmetric tensors on $\mathcal{R}^{d}$.

The canonical inner products and corresponding norms on $\mathcal{R}^{d}$ and $\mathcal{S}^{d}$ are defined, respectively, as follows:

$$
\begin{array}{cc}
u \cdot v=u_{i} v_{i}, & \|v\|=(v \cdot v)^{1 / 2} \quad \forall u, v \in \mathcal{R}^{d}, \\
\sigma \cdot \tau=\sigma_{i j} \tau_{i j}, & \|\tau\|=(\tau \cdot \tau)^{1 / 2} \quad \forall \sigma, \tau \in \mathcal{S}^{d} .
\end{array}
$$

Everywhere in the sequel the indices $i$ and $j$ run between 1 and $d$; the summation convention over repeated indices is implied.

Let $\Omega \subset \mathcal{R}^{d}$ be open, connected and bounded with a Lipschitz boundary $\Gamma$ that is divided into three disjoint measurable parts $\Gamma_{1}, \Gamma_{2}$ and $\Gamma_{3}$ such that meas $\left(\Gamma_{1}\right)>0$. Let $L^{2}(\Omega)$ be the Lebesgue space of 2 -integrable functions and $W^{k, p}(\Omega)$ be the Sobolev space of functions whose weak derivatives of orders less than or equal to $k$ are $p$-integrable on $\Omega$. Let $T>0$ and let $I \doteq(0, T)$ be the bounded time interval of interest, $\bar{I} \doteq[0, T]$. We also introduce the following spaces $H^{s}(\Omega)=W^{s, 2}(\Omega)$, " $H^{-s}$, denoted the dual space of $H^{s}$, and $C^{1}(\bar{I} ; X) \doteq\left\{v \in C(\bar{I} ; X) \mid v^{(1)} \in C(\bar{I} ; X)\right\}$.

Since the boundary is Lipschitz continuous, the outward unit normal vector which is denoted by $\nu$ exists a.e. on $\Gamma$. As the body is clamped on $\Gamma_{1}$, we know that the displacement field vanishes there. Surface traction of density $f_{2}$ acts on $\Gamma_{2}$ and a body force of density $f_{0}$ is applied in $\Omega$. The contact is bilateral, i.e., the normal displacement $u_{\nu}$ vanishes on $\Gamma_{3}$ at any time.

We introduce the spaces and the corresponding inner products as follows:

$$
H=\left\{v=\left(v_{1}, v_{2}, \ldots, v_{d}\right)^{T} \mid v_{i} \in L^{2}(\Omega), 1 \leq i \leq d\right\}=L^{2}(\Omega)^{d},
$$




$$
\begin{aligned}
& Q=\left\{\tau=\left(\tau_{i j}\right) \mid \tau_{i j}=\tau_{j i} \in L^{2}(\Omega), 1 \leq i, j \leq d\right\}=L^{2}(\Omega)_{s}^{d \times d}, \\
& Q_{1}=\{\tau \in Q \mid \operatorname{Div} \tau \in H\}, \\
& H_{1}=\left\{v=\left(v_{1}, v_{2}, \ldots, v_{d}\right)^{T} \mid v_{i} \in H^{1}(\Omega), 1 \leq i \leq d\right\}=H^{1}(\Omega)^{d}, \\
& V=\left\{v \in H_{1} \mid v=0 \text { on } \Gamma_{1}\right\}, \quad V_{1}=\left\{v \in V \mid v_{\nu}=0 \text { on } \Gamma_{3}\right\} .
\end{aligned}
$$

It is easy to check that the spaces $H, Q, H_{1}$ and $Q_{1}$ are all Hilbert spaces equipped with the inner products

$$
\begin{aligned}
& (u, v)_{H}=\int_{\Omega} u_{i}(x) v_{i}(x) d x, \quad(\sigma, \tau)_{Q}=\int_{\Omega} \sigma_{i, j}(x) \tau_{i, j}(x) d x, \\
& (u, v)_{H_{1}}=(u, v)_{H}+(\varepsilon(u), \varepsilon(v))_{Q}, \\
& (\sigma, \tau)_{Q_{1}}=(\sigma, \tau)_{Q}+(\operatorname{Div} \sigma, \operatorname{Div} \tau)_{H},
\end{aligned}
$$

where $\varepsilon$ denotes the deformation operator defined by $\varepsilon(u)=\left(\varepsilon_{i, j}(u)\right)$ with $\varepsilon_{i, j}(u)=\frac{1}{2}\left(u_{i, j}+u_{j, i}\right)$ and Div denotes the divergence operator defined by Div $\sigma=\left(\sigma_{i j, j}\right)$ and recall that $\sigma_{i j, j}=\frac{\partial \sigma_{i j}}{\partial x_{j}}$. The associated norms on the spaces will be denoted by $\|\cdot\|_{H},\|\cdot\|_{Q},\|\cdot\|_{H_{1}}$ and $\|\cdot\|_{Q_{1}}$, respectively.

Since $V$ is a closed subspace of the space $H_{1}$ and meas $\left(\Gamma_{1}\right)>0$, Korn's inequality holds:

$$
\|\varepsilon(v)\|_{Q} \geq \iota\|v\|_{H_{1}} \quad \forall v \in V
$$

where $\iota$ denotes a positive constant depending only on $\Omega$ and $\Gamma_{1}$. We define the inner product $(\cdot, \cdot)_{V}$ and the norm $\|\cdot\|_{V}$ on $V$ by

$$
(u, v)_{V}=(\varepsilon(u), \varepsilon(v))_{Q}, \quad\|v\|_{V}=\|\varepsilon(v)\|_{Q} \quad \forall u, v \in V .
$$

It follows that $\|\cdot\|_{H_{1}}$ and $\|\cdot\|_{V}$ are equivalent norms on $V$ and so $\left(V,\|\cdot\|_{V}\right)$ is a real Hilbert space. It is easy to see that $V_{1}$ is also a real Hilbert space with the inner product of the space $V$ given by $(2.1)$.

For every element $v \in H_{1}$, we use the notation $v$ for the trace of $v$ on $\Gamma$ and denote by $v_{\nu}$ and $v_{\tau}$ the normal and the tangential components of $v$ on $\Gamma$ given by $v_{\nu}=v \cdot \nu$ and $v_{\tau}=v-v_{\nu} \nu$, respectively.

We also denote by $\sigma_{\nu}$ and $\sigma_{\tau}$ the normal and the tangential traces of a function $\sigma \in Q$, respectively. We recall that, if $\sigma$ is a regular function, e.g., $\sigma \in C^{1}(\bar{\Omega})_{s}^{d \times d}$, then $\sigma_{\nu}=(\sigma \nu) \cdot \nu, \sigma_{\tau}=\sigma \nu-\sigma_{\nu} \nu$ and the following Green's formula holds:

$$
(\sigma, \varepsilon(v))_{Q}+(\operatorname{Div} \sigma, v)_{H}=\int_{\Gamma} \sigma \nu \cdot v d a \quad \forall v \in H_{1} .
$$

Let $r$ and $T$ be constants satisfying $0<r<T$. Let $\mathfrak{B}$ be the Borel $\sigma$ algebra of the interval $[-r, 0]$ and $\mu(\cdot)$ be a given finite signed measure defined on $([-r, 0], \mathfrak{B})$. Zhu $[26]$ defined the time-delay operator $G$ as follows: For any $\mathfrak{h} \in L^{2}((-r, \infty) \times \Omega)^{d}$,

$$
(G \mathfrak{h})(t, x) \doteq \int_{-r}^{0} \mathfrak{h}(t+\theta, x) \mu(d \theta) \quad \text { a.e. }(t, x) \in(0, \infty) \times \Omega .
$$


In order for (2.2) to make sense, we always take a Borel correction of $\mathfrak{h}$ as the integrand.

The following lemma is a fundamental result for operator $G$ :

Lemma 1. [26] For $\mathfrak{h} \in L^{2}((-r, \infty) \times \Omega)^{d}$, we have $G \mathfrak{h} \in L^{2}((0, \infty) \times \Omega)^{d}$. Furthermore, for any $g \in L^{2}((0, \infty) \times \Omega)^{d}, 0 \leq s \leq+\infty$ and $0 \leq s_{0} \leq r$, the following inequality holds:

$$
\begin{aligned}
& \left|\int_{\Omega} d x \int_{0}^{s+s_{0}}(G \mathfrak{h}) \cdot g d t\right| \leq \frac{1}{2}|\mu|([-r, 0])^{2} \int_{\Omega} d x \int_{-r}^{s}\|\mathfrak{h}\|^{2} d t \\
& \quad+\frac{1}{2} \int_{\Omega} d x \int_{0}^{s+s_{0}}\|g\|^{2} d t+\frac{1}{2} \mu|([-r, 0])| \mu \mid\left(\left[-s_{0}, 0\right]\right) \int_{\Omega} d x \int_{-r}^{s+s_{0}}\|\mathfrak{h}\|^{2} d t .
\end{aligned}
$$

Remark 1. Letting $s_{0}=0$ and $g=G \mathfrak{h}$ in Lemma 1 , it is easy to know that

$$
\|G\| \leq|\mu|([-r, 0]) \text {. }
$$

Now we take the contact problem into account. Based on (2.2), for $h \in$ $L^{2}((-r, \infty) \times \Omega)_{\mathcal{S}}^{d \times d}$, we derive the time-delay operator $\mathcal{G}$ of the form

$$
(\mathcal{G} h)(t, \varepsilon(u)) \doteq \int_{-r}^{0} h(t+\theta, \varepsilon(u)) \mu(d \theta) \quad \text { a.e. }(t, \varepsilon(u)) \in(0, \infty) \times \mathcal{S}^{d} \text {. }
$$

Remark 2. It is not hard to see that for any $h \in L^{2}((-r, \infty) \times \Omega)_{\mathcal{S}}^{d \times d}$, G $h$ in $L^{2}((0, \infty) \times \Omega)_{\mathcal{S}}^{d \times d}$ is independent of the choices of Borel corrections for $h$.

Remark 3. The simplest time-delay operator is the following discrete-type operator,

$$
G(t, y(\cdot))=\left(y\left(t-\delta_{1}\right), \ldots, y\left(t-\delta_{k}\right)\right), \quad \forall t \in I .
$$

Another common time-delay operator is the integral-type operator which corresponds to the accumulation characteristics of memory in practical problems, such as

$$
G(t, y(\cdot))=\int_{t_{0}}^{t} y(s-\delta) \mu(d s), \quad \forall t \in I .
$$

Remark 4. Operator $\mathcal{G}$ defined by (2.3) covers many interesting cases such as finitely many or countably many discrete delays and finitely many distributed delays.

Example 1. Let $X=\left\{a_{1}, a_{2}, \ldots, a_{n}, \cdots\right\}$ and $\mathcal{L}=2^{X}$. Let

$$
\mu_{1}(A)=\sum_{a_{i} \in A} \mu_{1}\left(a_{i}\right)=\sum_{a_{i} \in A} p_{i}, \quad \forall A \in \mathcal{L},
$$

where $\mu_{1}\left(a_{i}\right)=p_{i} \in \mathcal{R}^{+}$for $i=1,2, \ldots$ with $\mu_{1}(\emptyset)=0$. Then it is easy to see that

$$
(\mathcal{G} h)(t, \varepsilon(x))=\int_{-r}^{0} h(t+\theta, \varepsilon(x)) \mu_{1}(d \theta)=\sum_{a_{i} \in(-r, 0)} p_{i} h\left(t+a_{i}, \varepsilon(x)\right),
$$

which can be used to describe the countably many discrete delays. 
Example 2. Contact problems involving viscoelastic materials with long memory are a class of important problems, which have been studied by many authors, such as in $[4,10]$. For more details on the long memory models, we refer to $[13,20]$. Then

Let $\Omega=\mathcal{R}, \mathcal{L}$ be a $\sigma$-algebra of $\Omega, m$ be a Lebesgue measure and $f \equiv 1$.

$$
\mu(A)=\int_{A} 1 d m=A, \quad A \in \mathcal{L} .
$$

With variable substitution $s=-\theta$, the time-delay operator defined by $(2.3)$ will turn to be the form of $(\mathcal{G} h)(t, \varepsilon(u))=\int_{0}^{r} h(t-s, \varepsilon(u)) d s$. It implies that the long memory operator is a special case of the time-delay operator for $r=t$, $\mu(A)=A$ and the operator $h(t-s, \varepsilon(u))=\mathcal{C}(t-s) \varepsilon(u(s))$.

\section{The Model and the Primal Variational Formulation}

We assume that the contact is bilateral, and model the friction with a nonlocal version of Coulomb's law involving a normal regularization operator $\mathcal{T}$ : $H^{-\frac{1}{2}}(\Gamma) \rightarrow L^{2}(\Gamma)$.

Under the previous assumptions, the formulation of the frictional contact problem is as follows: Find a displacement field $u: \Omega \times I \rightarrow \mathcal{R}^{d}$ and a stress field $\sigma: \Omega \times I \rightarrow \mathcal{S}^{d}$ such that

$$
\begin{aligned}
& \sigma=\mathcal{A}(t, \varepsilon(\dot{u}(t)))+\mathcal{B}(t, \varepsilon(u(t)))+\mathcal{G} h(t, \varepsilon(u)) \quad \text { in } \Omega \times I, \\
& \text { Div } \sigma+f_{0}=0 \quad \text { in } \Omega \times I \text {, } \\
& u=0 \quad \text { on } \Gamma_{1} \times I \text {, } \\
& \sigma \nu=f_{1} \quad \text { on } \Gamma_{2} \times I \text {, } \\
& u_{\nu}=0, \quad\left\|\sigma_{\tau}\right\| \leq \alpha p\left(t,\left|\mathcal{T} \sigma_{\nu}\right|\right), \\
& \left\|\sigma_{\tau}\right\|<\alpha p\left(t,\left|\mathcal{T} \sigma_{\nu}\right|\right) \Rightarrow \dot{u}_{\tau}=0 \quad \text { on } \Gamma_{3} \times I, \\
& \left\|\sigma_{\tau}\right\|=\alpha p\left(t,\left|\mathcal{T} \sigma_{\nu}\right|\right) \Rightarrow \exists \lambda \geq 0 \quad \text { s.t. } \sigma_{\tau}=-\lambda \dot{u}_{\tau} . \\
& u(0)=u_{0} \quad \text { in } \Omega .
\end{aligned}
$$

The condition (3.1) represents the viscoelastic constitutive law in which $\mathcal{A}$, $\mathcal{B}$ and $\mathcal{G}$ are given nonlinear operators, called the viscosity operator, elasticity operator and time-delay operator, respectively. Note that the explicit dependence of the viscosity, elasticity and time-delay operators $\mathcal{A}, \mathcal{B}$ and $\mathcal{G}$ with respect to the time variable allows one to model more general situations when the properties of the material depend on the temperature, which plays the role of a parameter, i.e., its evolution in time is prescribed. Equality (3.2) represents the equilibrium equation. Conditions (3.3) and (3.4) are the displacement and traction boundary conditions, respectively. The condition (3.5) describes a frictional bilateral contact process, in which $p$ is a nonnegative valued function and $\alpha \geq 0$ is the coefficient of friction. Since the trace of the stress tensor on the boundary is too rough to be defined in the ordinary sense, we derive the nonlocal smoothing operator $\mathcal{T}: H^{-\frac{1}{2}}(\Gamma) \rightarrow L^{2}(\Gamma)$. Using the continuity of 
the regularization operator $\mathcal{T}$ and the continuity of the normal trace mapping $\xi \mapsto \xi_{\nu}: Q_{1} \rightarrow H^{-\frac{1}{2}}(\Gamma)$, we know that there exists a constant $C_{\mathcal{T}}>0$ such that

$$
\left\|\mathcal{T} \xi_{\nu}\right\|_{L^{2}\left(\Gamma_{3}\right)} \leq C_{\mathcal{T}}\|\xi\|_{Q_{1}} \quad \forall \xi \in Q_{1},
$$

where $C_{\mathcal{T}}$ relies on $\Omega, \Gamma_{1}, \Gamma_{3}$ and $\mathcal{T}$. In (3.6), $u_{0}$ is the initial displacement.

In the following we provide an elementary example of the mechanical problem, in which the constitutive law equation (3.2) holds.

In order to study the mechanical problem defined by (3.1)-(3.6), we assume that $\mathcal{A}, \mathcal{B}, h$ and $p$ satisfy the following conditions.

$\mathbf{H}(\mathcal{A}): \mathcal{A}: \Omega \times I \times \mathcal{S}^{d} \rightarrow \mathcal{S}^{d}$ is an operator such that

(i) $\left\|\mathcal{A}\left(x, t_{1}, \varepsilon_{1}\right)-\mathcal{A}\left(x, t_{2}, \varepsilon_{2}\right)\right\|_{Q} \leq L_{\mathcal{A}}\left(\left|t_{1}-t_{2}\right|+\left\|\varepsilon_{1}-\varepsilon_{2}\right\|\right)$ for all $t_{1}, t_{2} \in$ $I, \varepsilon_{1}, \varepsilon_{2} \in \mathcal{S}^{d}, x \in \Omega$ with $L_{A}>0$

(ii) $\left(\left(\mathcal{A}\left(x, t, \varepsilon_{1}\right)-\mathcal{A}\left(x, t, \varepsilon_{2}\right)\right),\left(\varepsilon_{1}-\varepsilon_{2}\right)\right)_{Q} \geq M\left\|\varepsilon_{1}-\varepsilon_{2}\right\|_{Q}^{2}$ for all $\varepsilon_{1}, \varepsilon_{2} \in \mathcal{S}^{d}$, a.e. $(x, t) \in \Omega \times I$ with $M>0$;

(iii) For any $\varepsilon \in \mathcal{S}^{d},(x, t) \mapsto \mathcal{A}(x, t, \varepsilon)$ is measurable on $\Omega \times I$;

(iv) The mapping $(x, t) \mapsto \mathcal{A}(x, t, 0) \in L^{2}(\Omega \times \bar{I})^{d \times d}$.

$\mathbf{H}(\mathcal{B}): \mathcal{B}: \Omega \times I \times \mathcal{S}^{d} \rightarrow \mathcal{S}^{d}$ is an operator such that

(i) $\left\|\mathcal{B}\left(x, t, \varepsilon_{1}\right)-\mathcal{B}\left(x, t, \varepsilon_{2}\right)\right\|_{Q} \leq L_{\mathcal{B}}\left\|\varepsilon_{1}-\varepsilon_{2}\right\|_{Q}$ for all $\varepsilon_{1}, \varepsilon_{2} \in \mathcal{S}^{d}$, a.e. $(x, t) \in$ $\Omega \times I$ with $L_{\mathcal{B}}>0$

(ii) For any $\varepsilon \in \mathcal{S}^{d}, \quad(x, t) \mapsto \mathcal{B}(x, t, \varepsilon)$ is measurable on $\Omega \times I$;

(iii) The mapping $(x, t) \mapsto \mathcal{B}(x, t, 0) \in L^{2}(\Omega \times \bar{I})^{d \times d}$.

$\mathbf{H}(h): h: \Omega \times I \times \mathcal{S}^{d} \rightarrow \mathcal{S}^{d}$ is an operator such that

(i) $\left\|h\left(x, t, \varepsilon_{1}\right)-h\left(x, t, \varepsilon_{2}\right)\right\|_{Q} \leq L_{h}\left\|\varepsilon_{1}-\varepsilon_{2}\right\|_{Q}$ for all $\varepsilon_{1}, \varepsilon_{2} \in \mathcal{S}^{d}$, a.e. $(x, t) \in$ $\Omega \times I$ with $L_{4}>0$;

(ii) For any $\varepsilon \in \mathcal{S}^{d},(x, t) \mapsto h(x, t, \varepsilon)$ is measurable on $\Omega \times I$;

(iii) The mapping $(x, t) \mapsto h(x, t, 0) \in L^{2}(\Omega \times \bar{I})^{d \times d}$.

$\mathbf{H}(p): p: \Gamma_{3} \times I \times \mathcal{R} \rightarrow \mathcal{R}_{+}$is an operator such that

(i) $\left|p\left(x, t_{1}, u_{1}\right)-p\left(x, t_{2}, u_{2}\right)\right| \leq L_{p}\left(\left|t_{1}-t_{2}\right|+\left|u_{1}-u_{2}\right|\right)$ for all $t_{1}, t_{2} \in I$, $u_{1}, u_{2} \in \mathcal{R}, x \in \Omega$ with $L_{p}>0$; 
(ii) For any $u \in \mathcal{R},(x, t) \mapsto p(x, t, u)$ is measurable on $\Omega \times I$;

(iii) The mapping $(x, t) \mapsto p(x, t, 0) \in L^{2}\left(\Gamma_{3} \times \bar{I}\right)$.

We assume the force and traction densities have the smoothness

$$
f_{0} \in C\left(\bar{I} ; L^{2}(\Omega)^{d}\right), \quad f_{1} \in C\left(\bar{I} ; L^{2}\left(\Gamma_{2}\right)^{d}\right)
$$

and the coefficient of friction $\alpha$ has the properties

$$
\alpha \in L^{\infty}(\Omega), \quad \alpha \geq 0 \quad \text { a.e. on } \Gamma_{3} \text {. }
$$

Denote by $f(t)$ the element of $V_{1}$ given by

$$
(f(t), v)_{V}=\int_{\Omega} f_{0}(t) \cdot v d x+\int_{\Gamma_{2}} f_{1}(t) \cdot v d a
$$

for all $v \in V_{1}$ and $t \in \bar{I}$ and let $j: \bar{I} \times Q_{1} \times V_{1} \rightarrow \mathcal{R}$ be the functional defined by

$$
j(t, \tau ; v)=\int_{\Gamma_{3}} \alpha p\left(t,\left|\mathcal{T} \tau_{\nu}\right|\right)\left\|v_{\tau}\right\| d a
$$

Since $\mathcal{T} \sigma_{\nu}$ lies in $L^{2}(\Gamma)^{d}$, from the assumption $\mathrm{H}(p)$ and (3.8), it follows that the integral in (3.9) is well defined on $\bar{I} \times Q_{1} \times V_{1}$. In the rest of this paper we always assume that $u_{0} \in V_{1}$ and let $\mathcal{Q}_{1} \doteq L^{2}\left(\bar{I} ; Q_{1}\right)$.

When $u$ and $\sigma$ are sufficiently regular functions satisfying (3.1)-(3.5), we can get the variational formulation of the quasistatic contact problem defined by (3.1)-(3.6) as follows:

Problem 1. Find a displacement field $u: \bar{I} \rightarrow V_{1}$ and a stress field $\sigma: \bar{I} \rightarrow Q_{1}$ such that (3.1), (3.6) hold and for all $v \in V_{1}$, a.e. $t \in \bar{I}$,

$$
(\sigma(t), \varepsilon(v-\dot{u}(t)))_{Q}+j(t, \sigma(t) ; v)-j(t, \sigma(t) ; \dot{u}(t)) \geq(f(t), v-\dot{u}(t))_{V},
$$

which is called a quasistatic integro-differential variational inequality.

In order to get the solvability of Problem 1, we first consider the following elliptic variational inequality of the second kind as follows: Given $f \in X$, find $u \in V$ such that for all $v \in V_{1}$, a.e. $t \in \bar{I}$,

$$
(A(t, u(t)), v-u(t))_{V}+j(v)-j(u(t)) \geq(f(t), v-u(t))_{V},
$$

where the operator $A: I \times V \rightarrow V$ is defined by

$$
(A(t, u(t)), v-u(t))_{V}=(\mathcal{A}(t, \varepsilon(u(t))), \varepsilon(v-u(t)))_{Q} .
$$

Lemma 2. [24] Let $V$ be a Hilbert space. Assume that $H(\mathcal{A})$ holds and $j: V \rightarrow$ $\overline{\mathcal{R}}$ is a proper, convex and l.s.c. functional. Then for any $f \in V$, the variational inequality (3.11) has a unique solution. 
Lemma 3. (Gronwall's inequality) Assume that $f, g \in C[a, b]$ satisfy

$$
f(t) \leq g(t)+c \int_{a}^{t} f(s) d s \quad t \in[a, b]
$$

where $c>0$ is a constant. Then

$$
f(t) \leq g(t)+c \int_{a}^{t} g(s) e^{c(t-s)} d s \quad t \in[a, b] .
$$

Moreover, if $g$ is nondecreasing, then

$$
f(t) \leq g(t) e^{c(t-a)} \quad t \in[a, b] .
$$

\section{An Existence and Uniqueness Result}

In this section, we present an existence and uniqueness result for Problem 1. Throughout this section, we assume that $\mathrm{H}(\mathcal{A}), \mathrm{H}(\mathcal{B}), \mathrm{H}(g), \mathrm{H}(h)$ and $(3.8)$ hold.

Theorem 1. There exists $\alpha_{0} \doteq \frac{M}{L_{\mathcal{A}} L_{p} c_{0} C_{\mathcal{T}}}>0$ which depends only on $\Omega, \Gamma_{1}$, $\Gamma_{3}, \mathcal{A}$ and $\mathcal{T}$ such that Problem 1 has a unique solution $(u, \sigma)$, if $\|\alpha\|_{L^{\infty}\left(\Gamma_{3}\right)}<$ $\alpha_{0}$. Moreover, the solution satisfies

$$
u \in C^{1}\left(\bar{I} ; V_{1}\right), \quad \sigma \in C\left(\bar{I} ; Q_{1}\right) .
$$

The proof of Theorem 1 is based on fixed point arguments, and is carried out in several steps. Let $\eta \in \mathcal{Q}_{1}$ and $\xi \in \mathcal{Q}_{1}$ be arbitrarily given. We consider the following auxiliary variational problem.

Problem 2. Find a velocity field $v_{\eta \zeta \xi}: \bar{I} \rightarrow V_{1}$ and a stress field $\sigma_{\eta \zeta \xi}: \bar{I} \rightarrow Q_{1}$ such that (3.6) holds and

$$
\begin{aligned}
& \left(\sigma_{\eta \zeta \xi}(t), \varepsilon\left(v-v_{\eta \zeta \xi}(t)\right)\right)_{Q}+j(t, \xi(t) ; v)-j\left(t, \xi(t) ; v_{\eta \zeta \xi}(t)\right) \\
& \quad \geq\left(f(t), v-v_{\eta \zeta \xi}(t)\right)_{V}
\end{aligned}
$$

for all $v \in V_{1}$ and a.e. $t \in \bar{I}$, where

$$
\sigma_{\eta \zeta \xi}(t)=\mathcal{A}\left(t, \varepsilon\left(v_{\eta \zeta \xi}(t)\right)\right)+\eta(t)+\zeta(t) .
$$

Lemma 4. There exists a unique solution $v_{\eta \zeta \xi} \in C\left(\bar{I} ; V_{1}\right)$ of Problem 2.

Proof. Let $t \in \bar{I}$. It follows from Lemma 2 that Problem 2 is uniquely solvable. Let $v_{\eta \zeta \xi}(t) \in V_{1}$ be the unique solution of Problem 2. Now we show that $v_{\eta \zeta \xi}(t) \in C\left(\bar{I}, V_{1}\right)$. Suppose that $t_{1}, t_{2} \in \bar{I}$. For simplicity we write $v_{\eta \zeta \xi}\left(t_{i}\right)=$ $v_{i}, \eta\left(t_{i}\right)=\eta_{i}, \zeta\left(t_{i}\right)=\zeta_{i}, f\left(t_{i}\right)=f_{i}$ and $\xi\left(t_{i}\right)=\xi_{i}$ with $i=1,2$. Using (4.2),(4.3) for $t=t_{1}, t_{2}$, we have

$$
\begin{aligned}
& \left(\mathcal{A}\left(t_{1}, \varepsilon\left(v_{1}\right)\right), \varepsilon\left(v-v_{1}\right)\right)_{Q}+\left(\eta_{1}, \varepsilon\left(v-v_{1}\right)\right)_{Q}+j\left(t_{1}, \xi_{1} ; v\right)-j\left(t_{1}, \xi_{1} ; v_{1}\right) \\
& \quad \geq\left(f_{1}, v-v_{1}\right)_{V}
\end{aligned}
$$


and

$$
\begin{aligned}
& \left(\mathcal{A}\left(t_{2}, \varepsilon\left(v_{2}\right)\right), \varepsilon\left(v-v_{2}\right)\right)_{Q}+\left(\eta_{2}, \varepsilon\left(v-v_{2}\right)\right)_{Q}+j\left(t_{2}, \xi_{2} ; v\right)-j\left(t_{2}, \xi_{2} ; v_{2}\right) \\
& \quad \geq\left(f_{2}, v-v_{2}\right)_{V} .
\end{aligned}
$$

By adding two inequalities with $v=v_{2}$ in (4.4) and $v=v_{1}$ in (4.5), we get

$$
\begin{aligned}
& \left(\mathcal{A}\left(t_{1}, \varepsilon\left(v_{1}\right)\right)-\mathcal{A}\left(t_{2}, \varepsilon\left(v_{2}\right)\right), \varepsilon\left(v_{1}-v_{2}\right)\right)_{Q} \\
& \quad \leq\left(\zeta_{1}-\zeta_{2}, \varepsilon\left(v_{2}-v_{1}\right)\right)_{Q}+\left(\eta_{1}-\eta_{2}, \varepsilon\left(v_{2}-v_{1}\right)\right)_{Q} \\
& \quad+\left(f_{1}-f_{2}, v_{1}-v_{2}\right)_{V}+D\left(t_{1}, t_{2}, \xi_{1}, \xi_{2}, v_{1}, v_{2}\right),
\end{aligned}
$$

where

$$
D\left(t_{1}, t_{2}, \xi_{1}, \xi_{2}, v_{1}, v_{2}\right)=j\left(t_{1}, \xi_{1} ; v_{2}\right)-j\left(t_{1}, \xi_{1} ; v_{1}\right)+j\left(t_{2}, \xi_{2} ; v_{1}\right)-j\left(t_{2}, \xi_{2} ; v_{2}\right) .
$$

Thus, we have

$$
\begin{aligned}
& \left(\mathcal{A}\left(t_{1}, \varepsilon\left(v_{1}\right)\right)-\mathcal{A}\left(t_{1}, \varepsilon\left(v_{2}\right)\right), \varepsilon\left(v_{1}-v_{2}\right)\right)_{Q} \\
& \quad \leq\left(\eta_{1}-\eta_{2}, \varepsilon\left(v_{2}-v_{1}\right)\right)_{Q}+\left(\mathcal{A}\left(t_{2}, \varepsilon\left(v_{2}\right)\right)-\mathcal{A}\left(t_{1}, \varepsilon\left(v_{2}\right)\right), \varepsilon\left(v_{1}-v_{2}\right)\right)_{Q} \\
& \quad+\left(\zeta_{1}-\zeta_{2}, \varepsilon\left(v_{2}-v_{1}\right)\right)_{Q}+D\left(t_{1}, t_{2}, \xi_{1}, \xi_{2}, v_{1}, v_{2}\right)+\left(f_{1}-f_{2}, v_{1}-v_{2}\right)_{V} .
\end{aligned}
$$

In terms of hypotheses $\mathrm{H}(\mathcal{A})$ on $\mathcal{A}$, we get

$$
\left(\mathcal{A}\left(t_{1}, \varepsilon\left(v_{1}\right)\right)-\mathcal{A}\left(t_{1}, \varepsilon\left(v_{2}\right)\right), \varepsilon\left(v_{1}-v_{2}\right)\right)_{Q} \geq M\left\|v_{1}-v_{2}\right\|_{V}^{2},
$$

and

$$
\left\|\mathcal{A}\left(t_{1}, \varepsilon\left(v_{2}\right)\right)-\mathcal{A}\left(t_{2}, \varepsilon\left(v_{2}\right)\right)\right\|_{Q} \leq L_{\mathcal{A}}\left|t_{1}-t_{2}\right| .
$$

Constituting a trace operator $\gamma: V \rightarrow L^{2}\left(\Gamma_{3}\right)^{d}$ with $\gamma v=\left.v\right|_{\Gamma_{3}}$, since $\gamma$ is a linear continuous operator, it implies that there exists a constant $c_{0}>0$ such that

$$
\|v\|_{L^{2}\left(\Gamma_{3}\right)^{d}} \leq c_{0}\|v\|_{V} .
$$

It follows from (3.7), (3.9), (4.10) and $\mathrm{H}(p)$ that

$$
D\left(t_{1}, t_{2}, \xi_{1}, \xi_{2}, v_{1}, v_{2}\right) \leq c_{1}\left\|\xi_{1}-\xi_{2}\right\|_{Q}\left\|v_{1}-v_{2}\right\|_{V}+\frac{c_{1}}{C_{\mathcal{T}}}\left|t_{1}-t_{2}\right|\left\|v_{1}-v_{2}\right\|_{V}
$$

where $c_{1}=\|\alpha\|_{L^{\infty}\left(\Gamma_{3}\right)} c_{0} C_{\mathcal{T}} L_{p}$. By (4.7)-(4.9) and (4.11), we have

$$
\begin{aligned}
\left\|v_{1}-v_{2}\right\|_{V} \leq & \frac{1}{M}\left(\left\|f_{1}-f_{2}\right\|_{V}+\left\|\eta_{1}-\eta_{2}\right\|_{Q}+\left\|\zeta_{1}-\zeta_{2}\right\|_{Q}+c_{1}\left\|\xi_{1}-\xi_{2}\right\|_{Q}\right. \\
& \left.+\left(L_{\mathcal{A}}+\frac{c_{1}}{C_{\mathcal{T}}}\right)\left|t_{1}-t_{2}\right|\right),
\end{aligned}
$$

which implies $v_{\eta \xi} \in C\left(\bar{I} ; V_{1}\right)$. The proof is completed. 
In order to get the unique solution of Problem 1, we consider the operator $\Lambda_{\eta \zeta}: C\left(\bar{I} ; Q_{1}\right) \rightarrow C\left(\bar{I} ; Q_{1}\right)$ defined by

$$
\Lambda_{\eta \zeta} \xi=\sigma_{\eta \zeta \xi} \quad \forall \xi \in C\left(\bar{I} ; Q_{1}\right) .
$$

For any $\eta, \zeta \in C(\bar{I} ; Q)$ and any given $\widetilde{\xi} \in C(\bar{I} ; Q)$, we write $v_{\eta \zeta}=v_{\eta \zeta \widetilde{\xi}}$.

Let $u_{\eta \zeta}: \bar{I} \rightarrow V_{1}$ be the function given by

$$
u_{\eta \zeta}(t)=\int_{0}^{t} v_{\eta \zeta}(s) d s+u_{0} \quad \text { a.e. } t \in \bar{I} .
$$

Lemma 5. If $\|\alpha\|_{L^{\infty}\left(\Gamma_{3}\right)}<\alpha_{0}$, for any $\eta, \zeta \in C\left(\bar{I} ; Q_{1}\right)$, then the operator $\Lambda_{\eta \zeta}$ has a unique fixed point $\xi_{\eta \zeta} \in C\left(\bar{I} ; Q_{1}\right)$.

Proof. For any $\eta, \zeta \in C(\bar{I} ; Q)$ and $\xi_{1}, \xi_{2} \in C\left(\bar{I} ; Q_{1}\right)$. We denote by $v_{i}=v_{\eta \zeta \xi_{i}}$ the solution of Problem 2 with $\xi=\xi_{i}$ for $i=1,2$. We write $\sigma_{\eta \zeta \xi_{i}}=\sigma_{i}$. By using the similar arguments in obtaining (4.6), we deduce

$$
\left(\sigma_{1}(t)-\sigma_{2}(t), \varepsilon\left(v_{1}(t)-v_{2}(t)\right)\right)_{Q} \leq D\left(t ; \xi_{1}, \xi_{2}, v_{1}, v_{2}\right) \quad \text { a.e. } t \in \bar{I},
$$

where

$$
\begin{aligned}
D\left(t ; \xi_{1}, \xi_{2}, v_{1}, v_{2}\right)= & j\left(t, \xi_{1}(t) ; v_{2}(t)\right)-j\left(t, \xi_{1}(t) ; v_{1}(t)\right) \\
& +j\left(t, \xi_{2}(t) ; v_{1}(t)\right)-j\left(t, \xi_{2}(t) ; v_{2}(t)\right)
\end{aligned}
$$

By (4.3) and $\mathrm{H}(\mathcal{A})$,

$$
\left(\sigma_{1}(t)-\sigma_{2}(t), \varepsilon\left(v_{1}(t)-v_{2}(t)\right)\right)_{Q} \geq M\left\|v_{1}(t)-v_{2}(t)\right\|_{V}^{2} \quad \text { a.e. } t \in \bar{I} .
$$

Using (3.7), (3.9), (4.10) and $\mathrm{H}(p)$, we have

$$
D\left(t ; \xi_{1}, \xi_{2}, v_{1}, v_{2}\right) \leq c_{1}\left\|\xi_{1}(t)-\xi_{2}(t)\right\|_{Q}\left\|v_{1}(t)-v_{2}(t)\right\|_{V} \quad \text { a.e. } t \in \bar{I} .
$$

It follows from (4.14) and (4.15) that

$$
\left\|v_{1}(t)-v_{2}(t)\right\| \leq \frac{c_{1}}{M}\left\|\xi_{1}(t)-\xi_{2}(t)\right\|_{Q} \quad \text { a.e. } t \in \bar{I}
$$

and so for a.e. $t \in \bar{I}$

$$
\left\|\Lambda_{\eta \zeta} \xi_{1}(t)-\Lambda_{\eta \zeta} \xi_{2}(t)\right\|_{Q}=\left\|\sigma_{1}(t)-\sigma_{2}(t)\right\|_{Q} \leq c_{2}\left\|\xi_{1}(t)-\xi_{2}(t)\right\|_{Q}
$$

where $c_{2}=\frac{c_{1} L_{\mathcal{A}}}{M}$. Since $\|\alpha\|_{L^{\infty}\left(\Gamma_{3}\right)}<\alpha_{0}$, the operator $\Lambda_{\eta \zeta}$ is a contraction on the space $C\left(\bar{I} ; Q_{1}\right)$. Therefore, the operator $\Lambda_{\eta \zeta}$ has a unique fixed point $\xi_{\eta \zeta} \in C\left(\bar{I} ; Q_{1}\right)$. The proof is completed.

In what follows, for any $\eta \in \mathcal{Q}_{1}$ and any given $\zeta \in \mathcal{Q}_{1}$, we write $v_{\eta}=v_{\eta \zeta \xi_{\eta \zeta}}$. By $\Lambda_{\eta \zeta} \xi_{\eta \zeta}=\xi_{\eta \zeta}$ and (4.12), we have $\sigma_{\eta \zeta \xi_{\eta \zeta}}=\xi_{\eta \zeta}$. For any $\eta \in \mathcal{Q}_{1}$, let $u_{\eta}: \bar{I} \rightarrow V_{1}$ be the function given by

$$
u_{\eta}(t)=\int_{0}^{t} v_{\eta}(s) d s+u_{0} \quad \text { a.e. } t \in \bar{I} .
$$


In addition, we define the operator $\Lambda_{\zeta}: \mathcal{Q}_{1} \rightarrow \mathcal{Q}_{1}$ by

$$
\Lambda_{\zeta} \eta(t)=\mathcal{B}\left(t, \varepsilon\left(u_{\eta}(t)\right)\right) \quad \forall \eta \in \mathcal{Q}_{1} \text {, a.e. } t \in \bar{I} .
$$

Lemma 6. If $\|\alpha\|_{L^{\infty}\left(\Gamma_{3}\right)}<\alpha_{0}$, then the operator $\Lambda_{\zeta}$ has a unique fixed point $\eta^{*} \in \mathcal{Q}_{1}$.

Proof. For any $\eta_{1}, \eta_{2} \in \mathcal{Q}_{1}$, let $u_{i}=u_{\eta_{i}}, \xi_{\eta_{i} \zeta}=\xi_{i}, v_{i}=v_{\eta_{i}}$ and $\sigma_{\eta_{i} \zeta \xi_{\eta_{i} \zeta}}=\sigma_{i}$ with $i=1,2$. Using (4.1) and arguments similar to ones used in obtaining (4.6), for a.e. $t \in \bar{I}$

$$
M\left\|v_{1}(t)-v_{2}(t)\right\|_{V} \leq\left(1+c_{1}\right)\left\|\eta_{1}(t)-\eta_{2}(t)\right\|_{Q}+c_{1} L_{\mathcal{A}}\left\|v_{1}(t)-v_{2}(t)\right\|_{V}
$$

and so

$$
\left\|v_{1}(t)-v_{2}(t)\right\|_{V} \leq c_{3}\left\|\eta_{1}(t)-\eta_{2}(t)\right\|_{Q} \quad \text { a.e. } t \in \bar{I},
$$

where $c_{3}=\frac{1+c_{1}}{M-c_{1} L_{\mathcal{A}}}$.

For the operator $\Lambda_{\zeta}$ defined by (4.17), it follows from (4.16), (4.18) and $\mathrm{H}(\mathcal{B})$ that

$$
\begin{aligned}
& \left\|\Lambda_{\zeta} \eta_{1}(t)-\Lambda_{\zeta} \eta_{2}(t)\right\|_{Q}^{2}=\left\|\mathcal{B}\left(t, \varepsilon\left(u_{1}(t)\right)\right)-\mathcal{B}\left(t, \varepsilon\left(u_{2}(t)\right)\right)\right\|_{Q}^{2} \\
& \quad \leq\left(L_{\mathcal{B}}\left\|u_{1}(t)-u_{2}(t)\right\|_{V}\right)^{2} \leq c_{4} \int_{0}^{t}\left\|\eta_{1}(s)-\eta_{2}(s)\right\|_{Q}^{2} d s \quad \text { a.e. } t \in \bar{I},
\end{aligned}
$$

where $c_{4}=c_{3}^{2} L_{\mathcal{B}}^{2}$. Iterating the last inequality $p$ times, we obtain

$$
\left\|\Lambda_{\zeta}^{p} \eta_{1}(t)-\Lambda_{\zeta}^{p} \eta_{2}(t)\right\|_{Q}^{2} \leq \frac{c_{4}^{p} t^{p-1}}{(p-1) !} \int_{0}^{t}\left\|\eta_{1}(s)-\eta_{2}(s)\right\|_{Q}^{2} \text { ds a.e. } t \in \bar{I},
$$

which leads to

$$
\left\|\Lambda_{\zeta}^{p} \eta_{1}-\Lambda_{\zeta}^{p} \eta_{2}\right\|_{\mathcal{Q}_{1}} \leq \frac{c_{4}^{p} T^{p}}{p !}\left\|\eta_{1}-\eta_{2}\right\|_{\mathcal{Q}_{1}} .
$$

Since $\lim _{p \rightarrow \infty} \frac{c_{4}^{p} T^{p}}{p !}=0,(4.20)$ implies that, for $p$ large enough, a power $\Lambda_{\zeta}^{p}$ of $\Lambda_{\zeta}$ is a contraction. It follows from the Banach's fixed point theorem that there exists a unique element $\eta^{*} \in V_{1}$ such that $\Lambda_{\zeta}^{p} \eta^{*}=\eta^{*}$. It is clear that $\Lambda_{\zeta}^{p}\left(\Lambda_{\zeta} \eta^{*}\right)=\Lambda_{\zeta}\left(\Lambda_{\zeta}^{p} \eta^{*}\right)=\Lambda_{\zeta} \eta^{*}$ and so $\Lambda_{\zeta} \eta^{*}$ is also a fixed point of the operator $\Lambda_{\zeta}^{p}$. Therefore,

$$
\Lambda_{\zeta} \eta^{*}=\eta^{*} .
$$

This shows that $\eta^{*}$ is a fixed point of $\Lambda_{\zeta}$. The uniqueness of the fixed point of $\Lambda_{\zeta}$ results straightforward from the uniqueness of the fixed point of $\Lambda_{\zeta}^{p}$. The proof is completed.

In what follows, for any $\zeta \in \mathcal{Q}_{1}$, we write $v_{\zeta}=v_{\eta^{*} \zeta \xi_{\eta^{*} \zeta}}$. For $\eta^{*}$, it follows from (4.12) and Lemma 5 that

$$
\sigma_{\eta^{*} \zeta \xi_{\eta^{*} \zeta}}=\xi_{\eta^{*} \zeta} .
$$


For any $\zeta \in \mathcal{Q}_{1}$, let $u_{\zeta}: \bar{I} \rightarrow V_{1}$ be the function given by

$$
u_{\zeta}(t)=\int_{0}^{t} v_{\zeta}(s) d s+u_{0} \quad \text { a.e. } t \in \bar{I} .
$$

In addition, we define the operator $\Lambda: \mathcal{Q}_{1} \rightarrow \mathcal{Q}_{1}$ by

$$
\Lambda \zeta(t)=\mathcal{G h}\left(t, \varepsilon\left(u_{\zeta}\right)\right) \quad \forall \zeta \in \mathcal{Q}_{1} \text {, a.e. } t \in \bar{I} .
$$

Lemma 7. If $\|\alpha\|_{L^{\infty}\left(\Gamma_{3}\right)}<\alpha_{0}$, then the operator $\Lambda$ has a unique fixed point $\zeta^{*} \in \mathcal{Q}_{1}$.

Proof. For any $\zeta_{1}, \zeta_{2} \in \mathcal{Q}_{1}$, let $u_{i}=u_{\zeta_{i}}, \xi_{\eta^{*} \zeta_{i}}=\xi_{i}, v_{i}=v_{\zeta_{i}}$ and $\sigma_{\eta^{*}} \zeta \xi_{\eta^{*} \zeta_{i}}=\sigma_{i}$ with $i=1,2$. Using (4.1) and arguments similar to ones used in obtaining (4.18), we have

$$
\left\|v_{1}(t)-v_{2}(t)\right\|_{V} \leq c_{3}\left\|\zeta_{1}(t)-\zeta_{2}(t)\right\|_{Q} \quad \text { a.e. } t \in \bar{I} .
$$

For the operator $\Lambda$ defined in (4.23), by (4.22), (4.24) and $\mathrm{H}(h)$, one has

$$
\begin{aligned}
\left\|\Lambda \zeta_{1}(t)-\Lambda \zeta_{2}(t)\right\|_{Q}^{2} & =\left\|\int_{-r}^{0}\left[h\left(t+\theta, \varepsilon\left(u_{1}\right)\right)-h\left(t+\theta, \varepsilon\left(u_{2}\right)\right)\right] \mu(d \theta)\right\|_{Q}^{2} \\
& \leq\left(L_{h}|\mu|([-r, 0])\left\|u_{1}-u_{2}\right\|_{V}\right)^{2} \\
& \leq c_{5} \int_{0}^{t^{\prime}}\left\|\zeta_{1}(s)-\zeta_{2}(s)\right\|_{Q}^{2} d s \quad \text { a.e. } t \in \bar{I}
\end{aligned}
$$

where $c_{5}=\left(c_{3} L_{h}|\mu|([-r, 0])\right)^{2}$. By using the similar arguments for obtaining (4.21), we have $\Lambda \zeta^{*}=\zeta^{*}$. It is easy known that $\Lambda$ has a unique fixed point $\zeta^{*}$. The proof is completed.

Now we prove Theorem 1.

Proof of Theorem 1. Let $\eta^{*} \in \mathcal{Q}_{1}$ be the fixed point of $\Lambda_{\zeta}$ and $\zeta^{*} \in \mathcal{Q}_{1}$ be the fixed point of $\Lambda$. Let $u_{\eta^{*} \zeta^{*}} \in C^{1}(\bar{I} ; Q)$ be the function defined by (4.13) for $\eta=\eta^{*}, \zeta=\zeta^{*}$. It follows from $\dot{u}_{\eta^{*} \zeta^{*}}=v_{\eta^{*} \zeta^{*}}$ that

$$
\begin{aligned}
(\mathcal{A}(t & \left.\left., \varepsilon\left(v_{\eta^{*} \zeta^{*}}(t)\right)\right), \varepsilon\left(v-v_{\eta^{*} \zeta^{*}}(t)\right)\right)_{Q}+\left(\eta^{*}(t), \varepsilon\left(v-v_{\eta^{*} \zeta^{*}}(t)\right)\right)_{Q} \\
& +\left(\zeta^{*}(t), \varepsilon\left(v-v_{\eta^{*} \zeta^{*}}(t)\right)\right)_{Q}+j\left(t, \xi_{\eta^{*} \zeta^{*}}(t) ; v\right)-j\left(t, \xi_{\eta^{*} \zeta^{*}}(t) ; v_{\eta^{*} \zeta^{*}}(t)\right) \\
\geq & \left(f(t), v-v_{\eta^{*} \zeta^{*}}(t)\right)_{V} \quad \forall v \in V_{1} \text {, a.e. } t \in \bar{I}
\end{aligned}
$$

Now inequality (3.10) follows from (4.12), (4.17) and (4.23). Moreover, since (4.13) implies $u_{\eta^{*} \zeta^{*}}(0)=u_{0}$, we conclude that $u_{\eta^{*} \zeta^{*}}$ is a solution of Problem 1 .

Let $u_{1}, u_{2} \in C\left(\bar{I} ; V_{1}\right)$ be two solutions of Problem 1. Letting $v_{i}=\dot{u}_{i}$ and

$$
\sigma_{i}(t)=\mathcal{A}\left(t, \varepsilon\left(\dot{u}_{i}(t)\right)\right)+\mathcal{B}\left(t, \varepsilon\left(u_{i}(t)\right)\right)+\mathcal{G} h\left(t, \varepsilon\left(u_{i}\right)\right)
$$

for $i=1,2$, we have

$$
u_{i}(t)=\int_{0}^{t} v_{i}(s) d s+u_{0} \quad \text { a.e. } t \in \bar{I} .
$$


For a.e. $t \in \bar{I}$, by using the similar arguments for obtaining (4.6), we get

$$
\begin{aligned}
&(\mathcal{A}(t\left.\left., \varepsilon\left(v_{1}(t)\right)\right)-\mathcal{A}\left(t, \varepsilon\left(v_{2}(t)\right)\right), \varepsilon\left(v_{1}(t)-v_{2}(t)\right)\right)_{Q} \\
& \leq\left(\mathcal{B}\left(t, \varepsilon\left(u_{1}(t)\right)\right)-\mathcal{B}\left(t, \varepsilon\left(u_{2}(t)\right)\right), \varepsilon\left(v_{2}(t)-v_{1}(t)\right)\right)_{Q}+D\left(t ; v_{1}, v_{2}\right) \\
& \quad+\left(\mathcal{G} h\left(t, \varepsilon\left(u_{1}(t)\right)\right)-\mathcal{G} h\left(t, \varepsilon\left(u_{2}(t)\right)\right), \varepsilon\left(v_{2}(t)-v_{1}(t)\right)\right)_{Q}
\end{aligned}
$$

where

$$
\begin{aligned}
D\left(t ; v_{1}, v_{2}\right)= & j\left(t, \sigma_{1}(t) ; v_{2}(t)\right)-j\left(t, \sigma_{1}(t) ; v_{1}(t)\right)+j\left(t, \sigma_{2}(t) ; v_{1}(t)\right) \\
& -j\left(t, \sigma_{2}(t) ; v_{2}(t)\right) .
\end{aligned}
$$

Using (3.8), (3.9), (4.10) and $\mathrm{H}(p)$, we deduce that, for a.e. $t \in \bar{I}$,

$$
D\left(t ; v_{1}, v_{2}\right) \leq c_{1}\left\|\sigma_{1}(t)-\sigma_{2}(t)\right\|_{Q}\left\|v_{1}(t)-v_{2}(t)\right\|_{V} .
$$

Therefore, from the assumption $\mathrm{H}(\mathcal{A}), \mathrm{H}(\mathcal{B}), \mathrm{H}(h)$ and (4.13) and (4.26), we know that

$$
\left\|v_{1}(t)-v_{2}(t)\right\|_{V} \leq c_{6} \int_{0}^{t}\left\|v_{1}(s)-v_{2}(s)\right\|_{V} d s \quad \text { a.e. } t \in \bar{I},
$$

where $c_{6}=\left(1+c_{1}\right)\left(L_{\mathcal{B}}+L_{h}|\mu|([-r, 0])\right) /\left(M-c_{1} L_{\mathcal{A}}\right)$. Now (4.27) implies $v_{1}=v_{2}$ with an application of the Gronwall inequality. It follows from (4.13) that $u_{1}=u_{2}$. If $\|\alpha\|_{L^{\infty}\left(\Gamma_{3}\right)}<\alpha_{0}$, then $(u, \sigma)$ with $u=u_{\eta^{*} \zeta^{*}}$ and $\sigma=\sigma_{\eta^{*} \zeta^{*}} \xi_{\eta^{*} \zeta^{*}}$ is the unique solution of Problem 1 satisfying the regularity condition, which completes the proof.

Remark 5. When $\mathcal{G}=0$ and the viscosity and elasticity operators $\mathcal{A}$ and $\mathcal{B}$ are implicit time dependent, Theorem 1 reduces to Theorem 13.3 of [12]. Furthermore, Theorem 1 is also a generalization of Theorem 3.1 of [22].

\section{Dual Variational Formulation}

By defining the admissible stress fields set $\Sigma(t, \tau)$, the results presented in Section 4 can be extended to the study of the dual problem. Reformulating the contact model as a variational inequality for the displacements, we can get the weak solution for such kind of problems. However, in practice, the main interest lies in the contact stress and the distribution of the stress is more important than the displacements. For this reason, it is meaningful to straightforward derive and analyze a variational formulation of the contact problem in terms of the stress, that is, the so-called dual variational formulation associated with (3.6) and (3.10). We show the equivalence between the prime variational formulation and the dual variational formulation. By Theorem 1 we get the corresponding existence and uniqueness result.

We define the admissible stress fields set $\Sigma(t, \tau)$ as follows: for all $\tau \in Q$ and a.e. $t \in \bar{I}$,

$$
\Sigma(t, \tau)=\left\{\xi \in Q \mid(\xi, \varepsilon(v))_{Q}+j(t, \tau ; v) \geq(f(t), v)_{V}, \forall v \in V_{1}\right\} .
$$


By adding two inequalities with $v=2 \dot{u}(t)$ and $v=0$ in (3.10), we deduce

$$
(\sigma(t), \varepsilon(\dot{u}(t)))_{Q}+j(t, \sigma(t) ; \dot{u}(t))=(f(t), \dot{u}(t))_{V} \quad \text { a.e. } t \in \bar{I},
$$

which implies, for a.e. $t \in \bar{I}$

$$
\sigma(t) \in \Sigma(t, \sigma(t)), \quad(\tau-\sigma(t), \varepsilon(\dot{u}(t)))_{Q} \geq 0 \quad \forall \tau \in \Sigma(t, \sigma(t)) .
$$

Therefore, (3.1), (3.6) and (5.2) lead to the following dual variational formulation.

Problem 3. Find a displacement $u: \bar{I} \rightarrow V_{1}$ and a stress field $\sigma: \bar{I} \rightarrow Q_{1}$ such that (3.1), (3.6) hold and for a.e. $t \in \bar{I}$

$$
\sigma(t) \in \Sigma(t, \sigma(t)), \quad(\tau-\sigma(t), \varepsilon(\dot{u}(t)))_{Q} \geq 0 \quad \forall \tau \in \Sigma(t, \sigma(t)) .
$$

The following theorem shows that prime variational formulation (Problem 1) and the dual variational formulation (Problem 3) are equivalent.

Theorem 2. Let conditions $H(\mathcal{A}), H(\mathcal{B}), H(h), H(p)$ and (3.9) hold and assume $(u, \sigma)$ satisfies (4.1). Then, $(u, \sigma)$ is a solution of Problem 1 if and only if it is a solution of Problem 3.

Proof. Let $(u, \sigma) \in C^{1}\left(\bar{I} ; V \times Q_{1}\right)$. We only need to show the equivalence between $(3.10)$ and $(5.3)$.

(I) $(3.10) \Rightarrow(5.3)$. Choosing $v=2 \dot{u}(t)$ and $v=0$ in (3.10), we know that (5.1) holds. It follows from (3.10) and (5.1) that

$$
(\sigma(t), \varepsilon(v))_{Q}+j(t, \sigma(t) ; v) \geq(f(t), v)_{V} \quad \forall v \in V_{1}, \quad \text { a.e. } t \in \bar{I}
$$

which implies that $\sigma(t) \in \Sigma(t, \sigma(t))$. Letting $\tau \in \Sigma(t, \tau(t))$, we have

$$
(\tau, \varepsilon(\dot{u}(t)))_{Q}+j(t, \sigma(t) ; \dot{u}(t)) \geq(f(t), \dot{u}(t))_{V} \quad \text { a.e. } t \in \bar{I} .
$$

Subtracting (5.1) from (5.4), we obtain

$$
(\tau-\sigma(t), \varepsilon(\dot{u}(t)))_{Q} \geq 0, \quad \forall \tau \in \Sigma(t, \sigma(t)) \text {, a.e. } t \in \bar{I},
$$

which implies that (5.3) holds.

(II) $(5.3) \Rightarrow(3.10)$. The subdifferentiability of the function $j(t, \sigma(t) ; \cdot)$ : $V \rightarrow \mathcal{R}$ implies that there exists $\tilde{f}(t) \in V$ such that

$$
j(t, \sigma(t) ; v)-j(t, \sigma(t) ; \dot{u}(t)) \geq(\tilde{f}(t), v-\dot{u}(t))_{V} \quad \forall v \in V_{1} \text {, a.e. } t \in \bar{I} .
$$

It follows that

$$
\begin{aligned}
& (f(t)-\tilde{f}(t), v-\dot{u}(t))_{V}+j(t, \sigma(t) ; v)-j(t, \sigma(t) ; \dot{u}(t)) \geq(f(t), v-\dot{u}(t))_{V} \\
& \quad \forall v \in V_{1}, \text { a.e. } t \in \bar{I} .
\end{aligned}
$$

Choosing $v=2 \dot{u}(t)$ and $v=0$ in (5.5), we have

$$
(f(t)-\tilde{f}(t), \dot{u}(t))_{V}+j(t, \sigma(t) ; \dot{u}(t))=(f(t), \dot{u}(t))_{V} \quad \text { a.e. } t \in \bar{I},
$$


which implies that

$$
\varepsilon(f(t)-\tilde{f}(t)) \in \Sigma(t, \sigma(t)) .
$$

It follows from (2.1), (5.3) and (5.6) that

$$
(f(t)-\tilde{f}(t), \dot{u}(t))_{V} \geq(\sigma(t), \varepsilon(\dot{u}(t)))_{Q} \quad \text { a.e. } t \in \bar{I} .
$$

Adding $j(t, \sigma(t) ; \dot{u}(t))$ to (5.7) and taking (5.6) into account, we obtain

$$
(f(t), \dot{u}(t))_{V} \geq(\sigma(t), \varepsilon(\dot{u}(t)))_{Q}+j(t, \sigma(t) ; \dot{u}(t)) \quad \text { a.e. } t \in \bar{I} .
$$

Noting $\sigma(t) \in \Sigma(t, \sigma(t))$ and $\dot{u}(t) \in V$, we have

$$
(\sigma(t), \varepsilon(\dot{u}(t)))_{Q}+j(t, \sigma(t) ; \dot{u}(t)) \geq(f(t), \dot{u}(t))_{V} \quad \text { a.e. } t \in \bar{I}
$$

and

$$
(\sigma(t), \varepsilon(v))_{Q}+j(t, \sigma(t) ; v) \geq(f(t), v)_{V} \quad \forall v \in V_{1} \text {, a.e. } t \in \bar{I} .
$$

Therefore, (5.8)-(5.10) lead to the inequality (3.10), which completes the proof.

Corollary 1. Let conditions $\mathrm{H}(\mathcal{A}), \mathrm{H}(\mathcal{B}), \mathrm{H}(h), \mathrm{H}(p)$ and (3.9) hold and let $\alpha_{0}>0$ be defined as in Theorem 1. If $\|\alpha\|_{L^{\infty}\left(\Gamma_{3}\right)}<\alpha_{0}$, then Problem 3 has a unique solution $(u, \sigma)$ satisfying (4.1).

\section{Conclusions}

In this paper, we have introduced and studied a mathematical model which describes the frictional contact between a viscoelastic body and a foundation. The problem is quasistatic, the contact is bilateral and is associated to the Coulomb's law of dry friction. In our model, the material's behavior is described with a nonlinear viscoelastic constitutive law with time-delay, which is quite different from previous one. We have formulated the frictional contact problem as a quasistatic integro-differential variational inequality. By employing the Banach fixed point theorem and the variational inequality technique, we have proved an existence and uniqueness of the solution for the problem. We have also derived a dual formulation of the problem in term of stress and given an equivalence theorem.

Although giving numerical results is not the purpose of this paper, it is interesting and important to study the numerical analysis for the frictional contact problem. As future work, we intend to investigate the numerical approximation for the quasistatic variational inequality problem with some possible applications to various technical systems, such as electric, pneumatic, hydraulic networks, and chemical processes.

\section{Acknowledgements}

The authors are grateful to the editor and the referees for their valuable comments and suggestions. 


\section{References}

[1] I. Argatov. Sinusoidally-driven flat-ended indentation of time-dependent materials: Asymptotic models for low and high rate loading. Mechanics of Materials, 48:56-70, 2012. http://dx.doi.org/10.1016/j.mechmat.2011.12.010.

[2] M. Barboteu, J.R. Fernández and T.-V. Hoarau-Mantel. A class of evolutionary variational inequalities with applications in viscoelasticity. Math. Models Methods Appl. Sci., 15:1595-1617, 2005. http://dx.doi.org/10.1142/S0218202505000820.

[3] M. Barboteu, J.R. Fernández and Y. Ouafik. Numerical analysis of a frictionless viscoelastic piezoelectric contact problem. Math. Model. Numer. Anal., 42:667682, 2008. http://dx.doi.org/10.1051/m2an:2008022.

[4] M. Campo, J.R. Fernández and Á. Rodríguez-Arós. A quasistatic contact problem with normal compliance and damage involving viscoelastic materials with long memory. Appl. Numer. Math., 58:1274-1290, 2008. http://dx.doi.org/10.1016/j.apnum.2007.07.003.

[5] O. Chau, D. Motreanu and M. Sofonea. Quasistatic frictional problems for elastic and viscoelastic materials. Appl. Math., 47(4):341-360, 2002. http://dx.doi.org/10.1023/A:1021753722771.

[6] V. Comincioli. A result concerning a variational inequality of evolution for operators of first order in $t$ with retarded terms. Ann. Mat. Pura Appl., IV. Ser., 88:357-378, 1971.

[7] N. Costea and A. Matei. Contact models leading to variational-hemivariational inequalities. J. Math. Anal. Appl., 386(2):647-660, 2012. http://dx.doi.org/10.1016/j.jmaa.2011.08.025.

[8] M. Delost and C. Fabre. On abstract variational inequalities in viscoplasticity with frictional contact. J. Optim. Theory Appl., 133(2):131-150, 2007. http://dx.doi.org/10.1007/s10957-007-9176-2.

[9] Z. Denkowski, S. Migórski and A. Ochal. Existence and uniqueness to a dynamic bilateral frictional contact problem in viscoelasticity. Acta. Appl. Math., 94(3):251-276, 2006. http://dx.doi.org/10.1007/s10440-006-9079-5.

[10] I. Figueiredo and L. Trabucho. A class of contact and friction dynamic problems in thermoelasticity and in thermoviscoelasticity. Internat. J. Engrg. Sci., 33:4566, 1995. http://dx.doi.org/10.1016/0020-7225(94)E0042-H.

[11] W. Han and M. Sofonea. Evolutionary variational inequalities arising in viscoelastic contact problems. SIAM J. Numer. Anal., 38(2):556-579, 2000. http://dx.doi.org/10.1137/S0036142998347309.

[12] W. Han and M. Sofonea. Quasistatic Contact Problems in Viscoelasticity and Viscoplasticity. Stud. Adv. Math. American Mathematical Society/International Press, 2002.

[13] A. Kulig and S. Migórski. Solvability and continuous dependence results for second order nonlinear evolution inclusions with a Volterra-type operator. Nonlinear Anal., 75(13):4729-4746, 2012. http://dx.doi.org/10.1016/j.na.2012.03.023.

[14] Y. Li and M.Y. Xu. Hysteresis loop and energy dissipation of viscoelastic solid models. Meth. Time-depend Mater., 11(1):1-14, 2007. http://dx.doi.org/10.1007/s11043-007-9027-4.

[15] A. Matei and M. Sofonea. Variational Inequalities with Applications. Springer, New York, 2009. 
[16] S. Migórski, A. Ochal and M. Sofonea. An evolution problem in nonsmooth elasto-viscoplasticity. Nonlinear Anal., 71(12):2766-2771, 2009. http://dx.doi.org/10.1016/j.na.2009.06.024.

[17] S. Migórski, A. Ochal and M. Sofonea. History-dependent subdifferential inclusions and hemivariational inequalities in contact mechanics. Nonlinear Anal. Real World Appl., 12(6):3384-3396, 2011.

http://dx.doi.org/10.1016/j.nonrwa.2011.06.002.

[18] S. Migórski, A. Ochal and M. Sofonea. Nonlinear Inclusions and Hemivariational Inequalities-Models and Analysis of Contact Problems. Springer, New York, 2013.

[19] J.Y. Park, J.U. Jeong and Y.H. Kang. Optimal control of parabolic variational inequalities with delays and state constraint. Nonlinear Anal., 71(12):329-339, 2009. http://dx.doi.org/10.1016/j.na.2008.11.060.

[20] Á. Rodríguez-Arós, M. Sofonea and J. Via no. Numerical analysis of a frictional contact problem for viscoelastic materials with long-term memory. Adv. Mech. Math., 18:1-11, 2009.

[21] M. Shillor. Special issue on recent advances in contact mechanics. Math. Comput. Model., 28:4-8, 1998.

[22] M. Shillor and M. Sofonea. A quasistatic viscoelastic contact problem with friction. Internat. J. Eng. Sci., 38(14):1517-1533, 2000. http://dx.doi.org/10.1016/S0020-7225(99)00126-3.

[23] M. Shillor, M. Sofonea and J.J. Telega. Models and Analysis of Quasistatic Contact, volume 655 of Lect. Notes Phys. Springer, Berlin, Herdelberg, 2004.

[24] S.S. Yao and N.J. Huang. A quasistatic contact problem for viscoelastic materials with slip dependent friction and time-delay. Math. Probl. Eng., 2012, 2012. http://dx.doi.org/10.1155/2012/396745.

[25] J. Yong and L. Pan. Quasi-linear parabolic partial differential equations with delays in the highest-order spatial derivatives. J. Austral. Math. Soc., Ser. A, 54(2):174-203, 1993.

[26] S.W. Zhu. Optimal control of variational inequalities with delays in the highest order spatial derivatives. Acta Math. Sin. (Engl. Ser.), 22(2):607-624, 2006. http://dx.doi.org/10.1007/s10114-005-0688-0. 